by Kepler, Galileo, and Newton, and through the development of modern astronomy.

For many years, our country has suffered from a strong propensity in university studies toward law and humanities over all other disciplines: on the average, 30 per cent of students go to law school, and together with humanities students the total is near to 50 per cent; meanwhile, we find some 5 to 10 per cent in engineering, 3 to 4 per cent in chemistry, 3 to 5 per cent in architecture, and only 2 to 3 per cent in agronomy.

It is still too early, only two years after the implementation of this new astronomy methodology, to show statistical data to prove the influence of this renewed approach on vocational orientation. Perhaps it may be necessary to wait 10 to 15 years to see the evidence of that influence. But there are some very positive indications that let us affirm we are in the right path: during the first two years with the new astronomy curriculum, many astronomy clubs have sprung up around the whole country. We cannot be absolutely certain about the future of these young people, but we have evidence of their interest, their yearning for scientific knowledge. They are our hope; they are the ones who will be able to change the ratio of students in scientific vs. humanistic disciplines. And this is probably where the future economic and social development of our country comes into play.

\title{
PROGRAMS OF I.A.U. COMMISSION 46 FOR DEVELOPING COUNTRIES
}

\author{
Donat G. Wentzel \\ University of Maryland, College Park, Maryland 20742, U.S.A.
}

\section{International Schools for Young Astronomers (ISYA)}

ISYA typically last about three weeks and attract roughly twenty students at the Master of Science level. About half the students are from the host country, the others from neighboring countries. ISYA are initiated by astronomically developing countries and have been held in Venezuela, Nigeria, Egypt, Portugal, Yugoslavia, Indonesia, China, and others. Two or three central topics are chosen. For each topic, a suitable faculty member is invited to stay for the entire school. Other topics appear for enrichment and are chosen according to local expertise and availability of other visitors. The IAU provides the prestige of an international venture and seeks to garner local support not only for the ISYA but also for the longer-term increase in local astronomical capabilities. Financial support is provided both locally and by the IAU, when possible ultimately from UNESCO. Perhaps the greatest benefits of an ISYA are the resulting lasting acquaintances and scientific cooperations. 


\section{Visiting Lecturers Program (VLP)}

The VLP is designed to lead to a significant improvement in the astronomical capabilities of the host country. Primarily, lecturers offer a series of courses. The VLP in Peru has operated for three years, the VLP in Paraguay has just started, and a VLP in Nigeria has been postponed for technical reasons. J. Sahade identified the lecturers for Peru and Paraguay; it was considered essential that lectures be in Spanish. The IAU supports travel costs; living expenses are provided locally. A contract between the IAU and the host institution seeks to assure the continued support of astronomy in the host country, including the employment of astronomers trained during the VLP.

\section{THE VISITING LECTURERS PROGRAM AT SAN MARCOS UNIVERSITY}

María Luisa Aguilar $\mathrm{H}$.

Av. Arica 830, Lima 5, Peru

Professors and students of San Marcos University (USM) have found that the Visiting Lecturers Program (VLP) of the IAU has helped to improve the study of astronomy in Peru. The Peruvian people and I are profoundly grateful.

The VLP and the International Schools for Young Astronomers (ISYA) advised us, pointing our perspectives and capacity for work in the correct direction. I feel that both the VLP and the ISYA are noble expressions of the universal message of scientific collaboration, and are the voices of the highest altruism of the astronomers who participate in them.

With reference to the VLP, the state of astronomy education can be described in three parts:

\section{Before VLP}

In Peru, there were a few introductory courses, and there was a little research in celestial mechanics in the 1950's. There was and still is a solar observatory, but one that does not carry out research. There was some popularization of astronomy, but in spite of it, the local population was not familiar with astronomy. Under these circumstances, our most overwhelming task was to identify new methods and strategies. So we studied the local conditions and characteristics, and chose the most appropriate institution to begin teaching astronomy. Later, we would progress to the rest of the country.

ISYA involvement provided communication with top-level astronomers. In 1982, we formed a group: Seminario de Astronomia $y$ Astrofisica (SAA) which 